\title{
Correlation between serum miR-122 and myocardial damage and ventricular function in patients with essential hypertension
}

\author{
Liangguo Wang ${ }^{1}$, Huabing Chen ${ }^{2}$ \\ ${ }^{1}$ Department of Cardiology, the First Affiliated Hospital of Wenzhou Medical University, Wenzhou, China; ${ }^{2}$ Department of Radiology, Second \\ Clinical School of Medicine, Changjiang University, Jingzhou, China \\ Contributions: (I) Conception and design: H Chen; (II) Administrative support: Both authors; (III) Provision of study materials or patients: Both \\ authors; (IV) Collection and assembly of data: Both authors; (V) Data analysis and interpretation: Both authors; (VI) Manuscript writing: Both \\ authors; (VII) Final approval of manuscript: Both authors. \\ Correspondence to: Huabing Chen. Department of Radiology, Second Clinical School of Medicine, Changjiang University, Jingzhou 434000, China. \\ Email: chb568751@163.com.
}

Background: Myocardial damage and decreased ventricular function are risk factors leading to a bad prognosis in patients with essential hypertension (EH). MicroRNAs play important roles in myocardial function impairment in patients with hypertension. The purpose of our research was to investigate the correlation between serum miR-122 and myocardial damage and ventricular functions in EH patients.

Methods: The clinic data of EH patients (group A, n=60) and healthy individuals (group B, n=60) from December 2016 to December 2019 in our hospital were collected and analyzed. Serum miR-122, myocardial damage markers [B-type brain natriuretic peptide (BNP), homocysteine (Hcy), cardiac troponin T (c'TnT) and creatine kinase $\mathrm{MB}$ isoenzyme (CK-MB)] and cardiac function indicators [ejection fraction (EF), left ventricular septal thickness (IVST), left ventricular isovolumic relaxation time (IVRT), left ventricular enddiastolic diameter (LVEDD), left ventricular posterior wall thickness (LVPWT), and left ventricular endsystolic diameter (LVESD)] were assessed in both groups. The correlation between serum miR-122 and myocardial damage markers and ventricular function indicators was analyzed.

Results: (I) The mean serum miR-122 concentration in group A and group B was $6.86 \pm 1.23$ and $3.36 \pm 1.87 \mu \mathrm{mol} / \mathrm{L}$, respectively. The serum miR-122 concentration in group A was evidently increased compared with that in group B. (II) The levels of BNP, Hcy, cTnT, and CK-MB in the peripheral blood in group A were evidently increased compared with those in group $\mathrm{B}(\mathrm{P}<0.05)$. (III) EF and IVRT were evidently decreased in group $A$ compared with that in group $\mathrm{B}(\mathrm{P}<0.05)$. (IV) Serum miR-122 concentration was positively correlated with the myocardial damage markers BNP, Hcy, cTnT and CK-MB, and serum miR-122 concentration was negatively correlated with the ventricular function indicators EF and IVRT but not significantly correlated with other ventricular function indicators (IVST, LVEDD, LVPWT and LVESD).

Conclusions: The serum miR-122 concentration in EH patients was higher than that in healthy individuals, and miR-122 concentration was positively correlated with myocardial damage markers. Serum miR-122 level was negatively correlated with the ventricular function indicators EF and IVRT but was not significantly correlated with other ventricular function indicators (IVST, LVEDD, LVPWT, and LVESD).

Keywords: Essential hypertension (EH); microRNA-122 (miR-122); myocardial damage; ventricular function; markers

Submitted Oct 16, 2020. Accepted for publication Jul 19, 2021.

doi: $10.21037 /$ jtd-21-677

View this article at: https://dx.doi.org/10.21037/jtd-21-677

(C) Journal of Thoracic Disease. All rights reserved. 


\section{Introduction}

Essential hypertension (EH), with a high prevalence and complication rates, is a major global health challenge. Early diagnosis and treatment and effective control of hypertension are essential for the prevention of cardiovascular and cerebrovascular diseases. However, the molecular mechanisms leading to the etiologies and progression of EH remain unknown. For the last few years, the morbidity of $\mathrm{EH}$ is increasing, which may be due to improvements in living conditions and increases in the pace and stress of life. In particular, although the diagnostic, therapeutic, and control rates of EH in young people are lower than those in elderly people, the prevalence of $\mathrm{EH}$ in young people has increased $(1,2)$. $\mathrm{EH}$ is a complex disease, and it is imperative to diagnose $\mathrm{EH}$ at an early stage to forbid subsequent complications. MicroRNAs (miRNAs) a group of single-stranded RNAs with a length of approximately 22 nucleotides and without coding function. MiRNAs regulate the expression of target proteinencoding genes at the post transcriptional level. One miRNA could regulate many gene expression, and one gene could be inhibited by many miRNAs. MiRNAs constitute a very complex molecular regulatory network, which can regulate the physiological and pathological processes of various cardiovascular diseases, including hypertension. Previous studies have confirmed that miRNAs, as key regulatory molecules, can regulate a variety of physiological pathological processes of cells, including cell proliferation, cell differentiation, cell apoptosis, survival, activation and morphogenesis. MiRNAs have been shown to regulate the pathogenesis and progression of hypertension. MiRNAs can affect the renin-angiotensin-aldosterone axis, and the function of vascular endothelial cells and vascular smooth muscle. Regulation of miRNA expression level in cells is one of the potential new methods for the treatment of hypertension. The miRNAs in blood can be new biomarkers of $\mathrm{EH}$, and the study of miRNAs can provide support for the understanding of the pathogenesis of $\mathrm{EH}$ and provide reference for the research and development of new drugs for the treatment of $\mathrm{EH}$ (3). MiR-122 is a mammalian liverspecific miRNA that targets the 3 ' untranslated region of mRNA, thereby down-regulating the expression of highaffinity cationic amino acid transporter-1 (Cat-1) $(4,5)$. The endothelial production of nitric oxide depends on the substrate of Cat-1, L-arginine, which enhances the transport of high-affinity Cat-1. Therefore, miR-122 can regulate the transport of arginine by affecting the protein expression of high-affinity Cat-1, thereby affecting the synthesis of nitric oxide $(6,7)$. Endothelium-dependent nitric oxide-mediated impairment of vasodilation is an important feature of $\mathrm{EH}$ and may precede elevated blood pressure $(8,9)$. Elevated blood pressure can accelerate vascular aging, and early diagnosis and intervention of vascular aging can prevent and delay cardiovascular events (10). However, clinically, which blood pressure parameters are associated with myocardial damage and ventricular function is a controversial topic. The purpose of our research was to analyze the correlation between serum miR-122 and myocardial damage and ventricular function. We present the following article in accordance with the STROBE reporting checklist (available at https://dx.doi.org/10.21037/jtd-21-677).

\section{Methods}

\section{General information}

All eligible EH patients in our hospital from December 2016 to December 2019 were selected as study subjects (group $\mathrm{A}, \mathrm{n}=60$ ). In addition, healthy individuals who underwent a physical examination during the same time period and had no symptoms of EH were randomly selected as control group from a same age range and sex ratio to decrease bias (group $B, n=60$ ). All procedures performed in this study involving human participants were in accordance with the Declaration of Helsinki (as revised in 2013). This study was approved by the ethics committee of the First Affiliated Hospital of Wenzhou Medical University . All patients signed an informed consent form. Number of subjects, age, and blood pressure were similar between the 2 groups $(\mathrm{P}>0.05)$ (Table 1).

\section{Inclusion and exclusion criteria}

The inclusion criteria were as follows: met the diagnostic criteria for EH in Chinese Guidelines for the Prevention and Treatment of Hypertension 2010, i.e., under conditions without antihypertensive drugs, brachial artery systolic blood pressure (SBP) $\geq 140 \mathrm{mmHg}$ and/or diastolic blood pressure $(\mathrm{DBP}) \geq 90 \mathrm{mmHg}$ on $\geq 3$ occasions, either continuously or on different days; age between 28-65 years; signed the informed consent form for this study; regular physical examination performed in our hospital, with complete clinical data; and completion of the study. The exclusion criteria were as follows: secondary hypertension, history of diabetes, chronic arrhythmia, severe liver, 
Table 1 General information of the 2 groups $(\bar{x} \pm \mathrm{s})$

\begin{tabular}{|c|c|c|c|c|}
\hline Parameters & Group A & Group B & $t$ & $p$ \\
\hline Body weight (kg) & $67.66 \pm 12.76$ & $69.57 \pm 15.77$ & 0.208 & 0.867 \\
\hline HDL-C (mmol/L) & $1.17 \pm 0.25$ & $1.43 \pm 0.27$ & 2.889 & 0.459 \\
\hline HDL-C (mmol/L) & $2.24 \pm 1.65$ & $2.94 \pm 1.26$ & 1.934 & 0.698 \\
\hline
\end{tabular}

HDL-C, high density lipoprotein cholesterol.

kidney or hematological diseases, cardiovascular disease, systemic infection, connective tissue disease, malignancy, thyroid disease, severe depression, dementia, chronic inflammatory diseases, mental illness, drug use history; and noncompletion of the experiment.

\section{Main observation indicators}

Fasting blood samples were collected from all participants. All samples were stored at room temperature for at least 30 minutes to allow blood coagulation and then centrifuged at 2,000 $\mathrm{g}$ for 15 minutes. Serum was collected and stored at $-80{ }^{\circ} \mathrm{C}$ until use. An EK003-80 (Phoenix Pharmaceuticals Inc, Burlingame, CA, USA) was used to determine serum concentrations of indicators. The levels of miR-122, brain natriuretic peptide (BNP), homocysteine (Hcy) and cardiac troponin $\mathrm{T}(\mathrm{c} T \mathrm{Tn} \mathrm{T})$ were determined using an enzymelinked immunosorbent assay (ELISA). Chromatography with fluorescence detection was used to determine the level of creatine kinase $\mathrm{MB}$ isoenzyme (CK-MB). An echocardiogram was performed by the same cardiac sonographer using color Doppler ultrasound. Ejection fraction (EF), left ventricular septal thickness (IVST), left ventricular isovolumic diastolic time (IVRT), left ventricular end-diastolic diameter (LVEDD), left ventricular posterior wall thickness (LVPWT), and left ventricular end-systolic diameter (LVESD) were recorded.

\section{Statistical analysis}

SPSS 22.0 software was used for the data analyses. Measurement data are expressed as $\bar{x} \pm \mathbf{s}$. Comparisons between 2 groups were performed using the independent samples $t$ test. Count data are expressed as (\%). Correlations between serum miR-122 and myocardial damage markers and ventricular function was examined using the Pearson correlation test. $\mathrm{P}<0.05$ indicated a statistically significant difference.

\section{Results}

Comparison of serum miR-122 concentration between the 2 groups

The average serum miR-122 concentration in group A was $6.86 \pm 1.23 \mu \mathrm{mol} / \mathrm{L}$, and the average serum miR-122 concentration in group B was $3.36 \pm 1.87 \mu \mathrm{mol} / \mathrm{L}$. The serum miR-122 concentration in group A was significantly higher than that in group $\mathrm{B}(t=8.304, \mathrm{P}<0.05)$.

\section{Comparison of myocardial damage markers between the 2 groups}

The comparison of the myocardial damage markers in the 2 groups indicated that the levels of BNP, Hcy, cTnT and CK-MB in the peripheral blood of patients in group A were significantly higher than those in the peripheral blood of individuals in group $\mathrm{B}(\mathrm{P}<0.05)$ (Table 2).

\section{Comparison of ventricular function between the 2 groups}

The comparison of ventricular function between the 2 groups indicated that EF and IVRT were both significantly lower in group $\mathrm{A}$ than in group $\mathrm{B}(\mathrm{P}<0.05)$. There were no significant differences in IVST, LVEED, LVPWT, and LVESD between the 2 groups $(\mathrm{P}>0.05)$ (Table 3$)$.

\section{Correlation between serum miR-122 and myocardial damage and ventricular function}

The correlation analysis of serum miR-122 and myocardial damage and ventricular function showed that serum miR-122 concentration was positively correlated with the myocardial damage markers BNP, Hcy, cTnT and CK-MB $(r=0.845,0.384,0.537$, and $0.587, \mathrm{P}<0.05)$ and 
Table 2 Comparison of myocardial damage markers between the 2 groups $(\bar{x} \pm \mathrm{S})$

\begin{tabular}{lccccc}
\hline Group & Case number & BNP $(\mathrm{ng} / \mathrm{L})$ & Hcy $(\mu \mathrm{mol} / \mathrm{L})$ & $\mathrm{cTnT}(\mathrm{ng} / \mathrm{L})$ & $\mathrm{CK}-\mathrm{MB}(\mathrm{mmol} / \mathrm{L})$ \\
\hline Group A & 60 & $939.36 \pm 122.54$ & $57.98 \pm 12.35$ & $55.43 \pm 10.67$ & $54.32 \pm 9.76$ \\
Group B & 60 & $603.23 \pm 100.75$ & $35.64 \pm 9.56$ & $33.11 \pm 8.59$ & $22.56 \pm 8.84$ \\
$t$ & & 9.236 & 9.754 & 8.277 & 6.358 \\
P & $<0.05$ & $<0.05$ & $<0.05$ & $<0.05$ \\
\hline
\end{tabular}

BNP, brain natriuretic peptide; Hcy, homocysteine; cTnT, cardiac troponin T; CK-MB, creatine kinase MB isoenzyme.

Table 3 Comparison of ventricular function between the 2 groups $(x \pm s)$

\begin{tabular}{lcccccc}
\hline Group & EF $(\%)$ & IVST $(\mathrm{mm})$ & IVRT $(\mathrm{msec})$ & LVEDD $(\mathrm{mm})$ & LVPWT $(\mathrm{mm})$ & LVESD $(\mathrm{mm})$ \\
\hline Group A & $32.85 \pm 6.45$ & $12.39 \pm 3.43$ & $131.67 \pm 13.64$ & $55.69 \pm 5.97$ & $11.49 \pm 1.65$ & $34.34 \pm 5.98$ \\
Group B & $48.57 \pm 5.97$ & $12.08 \pm 3.09$ & $154.66 \pm 15.93$ & $54.34 \pm 5.38$ & $11.03 \pm 1.78$ & $35.78 \pm 4.45$ \\
$t$ & 4.248 & 0.375 & 6.857 & 0.348 & 0.368 & 0.408 \\
P & $<0.05$ & 0.934 & $<0.05$ & 0.467 & 0.874 & 0.651 \\
\hline
\end{tabular}

EF, ejection fraction; IVST, left ventricular septal thickness; IVRT, left ventricular isovolumic diastolic time; LVEDD, left ventricular end-diastolic diameter; LVPWT, left ventricular posterior wall thickness; LVESD, left ventricular end-systolic diameter.

negatively correlated with the cardiac function indicators EF and IVRT $(r=-0.384$ and $-0.00184, \mathrm{P}<0)$; there was no significant correlation with other ventricular function indicators (IVST, LVEDD, LVPWT, and LVESD).

\section{Discussion}

Hypertension is one of the leading causes of cardiovascular disease, including coronary artery disease, heart failure, chronic kidney disease, peripheral vascular disease and stroke (11). Among these affected organs and tissues, subendocardial tissues are firstly damaged by hypertension. Subendocardial damage has been started even in asymptomatic condition. Thus, the changes of subendocardial status could early predict the hypertension induced cardiac damage (12). In hypertension condition, subendocardial fibrosis is formed and the diastolic function is impaired (13).

$\mathrm{EH}$ is a chronic and progressive cardiovascular disease. The tissue damage already occurs in subclinical status. Microalbuminuria could be observed in subclinical status of $\mathrm{EH}$. The level of urinary albumin excretion is associated by blood pressure, 24h systolic values and left ventricular function (14). In the early stage of $\mathrm{EH}$, myocardial strain values have been decreased and could be an indicator and treatment target for $\mathrm{EH}$ (15). The left atrial function impair and stiffness are presented in EH patients (16). The left ventricular deformation is also reduced in $\mathrm{EH}$ patient (17). Thus, finding efficient diagnostic and prognostic indicators and developing effective treatment strategies are of great importance to prevent $\mathrm{EH}$ progression and cure $\mathrm{EH}$.

The pathophysiological mechanism of hypertension is very complex, which is a combination of environmental factors, genetic factors and other factors. Although numerous studies have been conducted, the molecular mechanism is still not fully understood. In addition, continuously elevated blood pressure manifests as pathological changes in organs throughout the entire body, i.e., target organ damage (18-21).

MiRNAs are small noncoding RNA molecules with a length of approximately 22 nucleotides that act as posttranscriptional regulators of gene expression (22). The exact mechanism by which miRNAs induce translational inhibition or mRNA degradation remains unclear. Biologically, a single miRNA can target multiple genes, and a single gene can be targeted by multiple miRNAs. Therefore, miRNAs constitute a complex regulatory network that controls the physiological and pathological processes of a variety of cardiovascular diseases, including hypertension. Researchers have focused on circulating miRNAs as novel biomarkers of $\mathrm{EH}$, and new insights into pathology have been brought through the study of miRNAs. In 2008, researchers 
confirmed that miRNAs were found in human serum and plasma samples (23). They have since been detected in a wide range of biological fluids, including urine, saliva and cerebrospinal fluid (24). Circulating miRNAs have many features: they are very stable in blood, their concentrations are within the range detectable by current technology, and they often show tissue-specific expression; in addition, before the physical symptoms of a disease appear, miRNAs are likely to become dysfunctional (24). A previous study screened 1,700 miRNAs in plasma from 13 hypertension patients and 5 controls and identified 27 differentially expressed miRNAs, among which 14 were verified by quantitative polymerase chain reaction (qPCR) (25). After validation in the cohort, the following were confirmed: the expression of HCMVencoded miRNA (hmcv-miR-UL112) in hypertension patients was 2.5 times that in the healthy control group, and HCMV seropositivity and miR-UL122 expression were both independently associated with an increased risk of hypertension (26). Recently, researchers used microarrays to screen approximately 1,350 miRNAs in 6 hypertension patients and 6 healthy individuals. They found that compared with that in the control group, the expression of miR-122, miR-425 and miR-505 was higher in hypertension patients. This result is consistent with those in this study, i.e., miR-122 expression was significantly higher in $\mathrm{EH}$ patients than in the control group. This result indicates that miR-122 can be used as a biomarker for hypertension.

Serum BNP, Hcy, cTnT and CK-MB are commonly used markers of myocardial damage, reflecting the presence and degree. An increase in the expression of myocardial damage markers is an independent risk factor for cardiovascular disease in $\mathrm{EH}$ patients, consistent with the results of this study, which indicated that serum BNP, Hcy, cTnT and CK-MB levels in EH patients all increased. Increased cTnT expression indicated ongoing myocardial cell injury. cTnT can serve as an good indicator for cardiovascular and cerebrovascular events in EH patients (27). The decreased cTnT level is associated with left ventricular ejection fraction and left atrial volume index restoration and left atrium and ventricular remodeling (27). c'TnT is negative correlated with left ventricular mass index. cTnT is strongly associated with subclinical left ventricular hypertrophy and function (28), and could be an indicator for hypertrophy and fibrosis in hypertrophic cardiomyopathy and hypertensive patients (29,30).

In addition, the results of the Pearson correlation test indicated that serum miR-122 in EH patients was positively correlated with myocardial damage markers. These results indicated that compared with those in the healthy controls, the levels of myocardial damage markers in $\mathrm{EH}$ patients were significantly abnormal, suggesting the presence of myocardial damage in $\mathrm{EH}$ patients. Studies have shown that changes in cardiac structure and function in the disease course of $\mathrm{EH}$ not only directly affect the prognosis of patients but also indirectly lead to disease progression. Therefore, it is very important to clarify the impact of cardiac structure and function on EH. In this study, color Doppler ultrasound was used for echocardiography, and EF, IVST, IVRT, LVEDD, LVPWT, and LVESD were recorded. The results showed that both EF and IVRT were significantly lower in $\mathrm{EH}$ patients than in the control group. There was no significant difference between the 2 groups in terms of IVST, LVEED, LVPWT, and LVESD. Pearson's correlation test showed that serum miR-122 was negatively correlated with the ventricular function indicators EF and IVRT in EH patients but not significantly correlated with other ventricular function indicators (IVST, LVEDD, LVPWT, and LVESD). Further investigation is needed to explore the reasons for these findings.

MiR-122 has been proved to be a good indicator of heart failure and cardiovascular injury. MiR-122-5p could inhibit the collagen synthesis and differentiation of cardiac fibroblasts via targeting HIF-1 $\alpha$ (31). The severity of cardiovascular disease, including atherosclerosis, AMI, heart failure, atrial fibrillation and hypertension, is related to the expression of miR-122 expression. MiR-122 is also a risk factor of these cardiovascular diseases (32). MiR-122 could regulate autophagy, apoptosis, fibrosis, inflammatory reaction and dysfunction in cardiovascular system (33-36). MiR122 could regulate apoptosis-autophagy balance of aortic adventitial fibroblasts in angiotensin II induced hypertension via targeting SIRT6-elabela-ACE2 pathway (33). MiR-122 knockout could reduce inflammatory response and tissue injury via TLR7 pathway (37). MiR-122 inhibitor contributes to reduce lipid accumulation and inflammatory reaction via inhibiting TR4/MyD88/NF- $\kappa B$ pathway (38). In this study, we confirmed miR-122 expression was correlated to ventricular function. The target genes and pathways in this model needs to be further studied.

As miRNAs have been involved in $\mathrm{EH}$ progression, thus, miRNAs could be a prognostic indicator in $\mathrm{EH}$ patients (39). Some miRNAs, like miR-122 and miR575 , were stable upregulated in EH patients and could be diagnostic and prognostic indicators. MiR-9 and miR-126 were stable decreased in the serum of $\mathrm{EH}$ patients, which could also be a prognostic indicator (40). In EH patients, 
platelet miRNAs, like miR-22 and miR-223, are also strong prognostic indicator for $\mathrm{EH}$ patients (41).

However, there were some limitations in this study. The number of patient samples was small. The direct target gene of miR-122 was not verified in animal or cell experiments. The regulation of miR-122 on the cardiac fibroblast and myocardial cell needed to be further studied.

In summary, the serum miR-122 concentration in $\mathrm{EH}$ patients was higher than that in healthy individuals, and miR-122 concentration was positively correlated with myocardial damage markers. Serum miR-122 concentration was positively correlated with the ventricular function indicators EF and IVRT but not significantly correlated with other ventricular function indicators (IVST, LVEDD, LVPWT, and LVESD).

\section{Acknowledgments}

Funding: None.

\section{Footnote}

Reporting Checklist: The authors have completed the STROBE reporting checklist. Available at https://dx.doi. org/10.21037/jtd-21-677

Data Sharing Statement: Available at https://dx.doi. org/10.21037/jtd-21-677

Conflicts of Interest: Both authors have completed the ICMJE uniform disclosure form (available at https://dx.doi. org/10.21037/jtd-21-677). The authors have no conflicts of interest to declare.

Ethical Statement: The authors are accountable for all aspects of the work in ensuring that questions related to the accuracy or integrity of any part of the work are appropriately investigated and resolved. All procedures performed in this study involving human participants were in accordance with the Declaration of Helsinki (as revised in 2013). This study was approved by the ethics committee of the First Affiliated Hospital of Wenzhou Medical University. All patients voluntarily signed an informed consent form.

Open Access Statement: This is an Open Access article distributed in accordance with the Creative Commons Attribution-NonCommercial-NoDerivs 4.0 International License (CC BY-NC-ND 4.0), which permits the non- commercial replication and distribution of the article with the strict proviso that no changes or edits are made and the original work is properly cited (including links to both the formal publication through the relevant DOI and the license). See: https://creativecommons.org/licenses/by-nc-nd/4.0/.

\section{References}

1. Gkaliagkousi E, Gavriilaki E, Triantafyllou A, et al. Clinical Significance of Endothelial Dysfunction in Essential Hypertension. Curr Hypertens Rep 2015;17:85.

2. Gupta-Malhotra M, Shete S, Barratt MS, et al. Epidemiology of Childhood Onset Essential Hypertension. J Hum Hypertens 2018;32:808-13.

3. Brater DC. Benefits and risks of torasemide in congestive heart failure and essential hypertension. Drug Saf 1996;14:104-20.

4. Marques FZ, Booth SA, Charchar FJ. The emerging role of non-coding RNA in essential hypertension and blood pressure regulation. J Hum Hypertens 2015;29:459-67.

5. Zhong F, Zhuang L, Wang Y, et al. Homocysteine levels and risk of essential hypertension: A meta-analysis of published epidemiological studies. Clin Exp Hypertens 2017;39:160-7.

6. Monticone S, D'Ascenzo F, Moretti C, et al. Cardiovascular events and target organ damage in primary aldosteronism compared with essential hypertension: a systematic review and meta-analysis. Lancet Diabetes Endocrinol 2018;6:41-50.

7. Goldkorn R, Yurenev A, Blumenfeld J, et al. Echocardiographic comparison of left ventricular structure and function in hypertensive patients with primary aldosteronism and essential hypertension. Am J Hypertens 2002;15:340-5.

8. Yang Y, Zhu LM, Xu JZ, et al. Comparison of left ventricular structure and function in primary aldosteronism and essential hypertension by echocardiography. Hypertens Res 2017;40:243-50.

9. Gupta-Malhotra M, Hamzeh RK, Poffenbarger T, et al. Myocardial Performance Index in Childhood Onset Essential Hypertension and White Coat Hypertension. Am J Hypertens 2016;29:379-87.

10. Ohno Y, Sone M, Inagaki N, et al. Prevalence of Cardiovascular Disease and Its Risk Factors in Primary Aldosteronism: A Multicenter Study in Japan. Hypertension 2018;71:530-7.

11. Zhang S, Liu L, Huang YQ, et al. The association between serum uric acid levels and ischemic stroke in essential 
hypertension patients. Postgrad Med 2020;132:551-8.

12. Lu S, Bao MY, Miao SM, et al. Prevalence of hypertension, diabetes, and dyslipidemia, and their additive effects on myocardial infarction and stroke: a cross-sectional study in Nanjing, China. Ann Transl Med 2019;7:436.

13. Beyhoff N, Lohr D, Foryst-Ludwig A, et al. Characterization of Myocardial Microstructure and Function in an Experimental Model of Isolated Subendocardial Damage. Hypertension 2019;74:295-304.

14. Mettimano M, Specchia ML, Migneco A, et al. Microalbuminuria as a marker of cardiac damage in essential hypertension. Eur Rev Med Pharmacol Sci 2001;5:31-6.

15. Liu H, Wang J, Pan Y, et al. Early and Quantitative Assessment of Myocardial Deformation in Essential Hypertension Patients by Using Cardiovascular Magnetic Resonance Feature Tracking. Sci Rep 2020;10:3582.

16. Huang J, Ni CF, Yang C, et al. Assessment of subclinical left atrial myocardial dysfunction in essential hypertension patients with normal left ventricle function by twodimensional strain and volume-derived variables. J Clin Ultrasound 2021;49:659-66.

17. Li XM, Peng LQ, Shi R, et al. Impact of gender on left ventricular deformation in patients with essential hypertension assessed by cardiac magnetic resonance tissue tracking. J Magn Reson Imaging 2021;53:1710-20.

18. Liu Y, Lin Y, Zhang MM, et al. The relationship of plasma renin, angiotensin, and aldosterone levels to blood pressure variability and target organ damage in children with essential hypertension. BMC Cardiovasc Disord 2020;20:296.

19. Zhou B, Li C, Shou J, et al. The cumulative blood pressure load and target organ damage in patients with essential hypertension. J Clin Hypertens (Greenwich) 2020;22:981-90.

20. Cai W, Lang M, Jiang X, et al. Correlation among high salt intake, blood pressure variability, and target organ damage in patients with essential hypertension: Study protocol clinical trial (SPIRIT compliant). Medicine (Baltimore) 2020;99:e19548.

21. El Mokadem M, Boshra H, Abd El Hady Y, et al. Correlation between blood pressure variability and subclinical target organ damage in patients with essential hypertension. J Hum Hypertens 2020;34:641-7.

22. Zhao L, Tian C, Xiao E, et al. Expression and regulatory network of miRNAs in head and neck squamous cell carcinoma. Transl Cancer Res 2020;9:4607-17.

23. Rahmel T, Schäfer ST, Frey UH, et al. Increased circulating microRNA-122 is a biomarker for discrimination and risk stratification in patients defined by sepsis-3 criteria. PLoS One 2018;13:e0197637.

24. $\mathrm{Pu} \mathrm{XX}$, Huang GL, Guo HQ, et al. Circulating miR-221 directly amplified from plasma is a potential diagnostic and prognostic marker of colorectal cancer and is correlated with p53 expression. J Gastroenterol Hepatol 2010;25:1674-80.

25. Khalyfa A, Kheirandish-Gozal L, Bhattacharjee R, et al. Circulating microRNAs as Potential Biomarkers of Endothelial Dysfunction in Obese Children. Chest 2016;149:786-800.

26. Zhang HG, Zhang QJ, Li BW, et al. The circulating level of miR-122 is a potential risk factor for endothelial dysfunction in young patients with essential hypertension. Hypertens Res 2020;43:511-7.

27. Setsuta K, Kitahara Y, Arae M, et al. Elevated cardiac troponin $\mathrm{T}$ predicts adverse outcomes in hypertensive patients. Int Heart J 2011;52:164-9.

28. Lyngbakken MN, Aagaard EN, Kvisvik B, et al. Cardiac Troponin I and T Are Associated with Left Ventricular Function and Structure: Data from the Akershus Cardiac Examination 1950 Study. Clin Chem 2020;66:567-78.

29. Askin L, Tasolar H, Aksu U, et al. Discerning the relationship between left ventricular geometry, highsensitivity troponin $\mathrm{T}$, and nondipper hypertension. Blood Press Monit 2018;23:12-8.

30. Gawor M, Śpiewak M, Kubik A, et al. Circulating biomarkers of hypertrophy and fibrosis in patients with hypertrophic cardiomyopathy assessed by cardiac magnetic resonance. Biomarkers 2018;23:676-82.

31. Feng W, Ying Z, Ke F, et al. Apigenin suppresses TGF$\beta 1$-induced cardiac fibroblast differentiation and collagen synthesis through the downregulation of HIF-1 expression by miR-122-5p. Phytomedicine 2021;83:153481.

32. Liu Y, Song JW, Lin JY, et al. Roles of MicroRNA-122 in Cardiovascular Fibrosis and Related Diseases. Cardiovasc Toxicol 2020;20:463-73.

33. Song JJ, Yang M, Liu Y, et al. MicroRNA-122 aggravates angiotensin II-mediated apoptosis and autophagy imbalance in rat aortic adventitial fibroblasts via the modulation of SIRT6-elabela-ACE2 signaling. Eur J Pharmacol 2020;883:173374.

34. Zhao Z, Zhong L, Li P, et al. Cholesterol impairs hepatocyte lysosomal function causing M1 polarization of macrophages via exosomal miR-122-5p. Exp Cell Res 2020;387:111738.

35. Filipowicz $W$, Grosshans $H$. The liver-specific microRNA 
miR-122: biology and therapeutic potential. Prog Drug Res 2011;67:221-38.

36. Gatfield D, Le Martelot G, Vejnar CE, et al. Integration of microRNA miR-122 in hepatic circadian gene expression. Genes Dev 2009;23:1313-26.

37. Wang Y, Liang H, Jin F, et al. Injured liver-released miRNA-122 elicits acute pulmonary inflammation via activating alveolar macrophage TLR7 signaling pathway. Proc Natl Acad Sci U S A 2019;116:6162-71.

38. Hu Y, Du G, Li G, et al. The miR-122 inhibition alleviates lipid accumulation and inflammation in NAFLD cell

Cite this article as: Wang L, Chen H. Correlation between serum miR-122 and myocardial damage and ventricular function in patients with essential hypertension. J Thorac Dis 2021;13(8):4999-5006. doi: 10.21037/jtd-21-677 model. Arch Physiol Biochem 2019:1-5.

39. Wise IA, Charchar FJ. Epigenetic Modifications in Essential Hypertension. Int J Mol Sci 2016;17:451.

40. Kontaraki JE, Marketou ME, Zacharis EA, et al. MicroRNA-9 and microRNA-126 expression levels in patients with essential hypertension: potential markers of target-organ damage. J Am Soc Hypertens 2014;8:368-75.

41. Marketou M, Kontaraki J, Papadakis J, et al. Platelet microRNAs in hypertensive patients with and without cardiovascular disease. J Hum Hypertens 2019;33:149-56. 